\title{
Both Senkyunolide I and Ligustrazine Suppress Retinal Spreading Depression
}

\author{
Li YL', Wang $Y^{1}$ and Wang $M^{1,2 *}$ \\ ${ }^{1}$ Centre for Neuroscience, Xi'an J iaotong- Liverpool \\ University (XJTLU), Suzhou, China \\ ${ }^{2}$ Department of Biological Sciences, XJTLU, Suzhou, \\ China \\ *Corresponding author: Minyan Wang, Department \\ of Biological Sciences, Xi'an J iaotong-Liverpool \\ University, Suzhou, 215123, China; Email: minyan. \\ wang@xjtlu.edu.cn
}

Received: March 13, 2017; Accepted: April 04, 2017; Published: April 10, 2017

\begin{abstract}
Senkyunolide I (SEI) and ligustrazine are two active components of Chuanxiong Rhizoma that is commonly prescribed for migraine therapy; However their actions in early stage of migraine are not fully understood. This study aimed to investigate effects of these two compounds in a chick migraine model and to explore their possible mechanism of action. An in vitro chick retinal spreading depression (RSD) was induced by $\mathrm{KCl}$ and intrinsic optical imaging was used for RSD recording. Enzyme-linked immunosorbent activity assay was used to quantify retinal Calcitonin Gene-Related Peptide (CGRP) level. The results show that SEI concentration-dependently suppressed both the magnitude and propagation rate of RSD in the chick retina. Similarly, but to a lesser extent, the magnitude of RSD was also suppressed by ligustrazine. ELISA data further shows that the retinal CGRP level during RSD was slightly reduced by SEl but this reduction did not reach significance. This data demonstrates that both SEI and ligustrazine had the ability in suppressing RSD, suggesting these two active components of Chuanxiong Rhizoma may perform similar function for migraine prophylaxis and their activity is of neuronal mechanism.
\end{abstract}

Keywords: Senkyunolide I; Ligustrazine; Retinal spreading depression; Migraine

\section{Abbreviations}

RSD: Retinal Spreading Depression; CGRP: Calcitonin GeneRelated Peptide; NO: Nitric Oxide; SEI: Senkyunolide I; CSD: Cortical Spreading Depression; MDA: Methylenedioxyamphetamine; SOD: Superoxide Dismutase; NMDAR: N-Methyl-D-Aspartate Receptor; ROS: Reactive Oxidative Stress; AOI: Area of Interest; AUC: Area Under the Curve

\section{Introduction}

Chuanxiong Rhizoma is the most commonly prescribed traditional Chinese medicine for migraine therapy [1] and ischemia [2]. The herb contains many active ingredients, which are classified into phthalide compounds, organic acids, phenols, ferulic acid, essential oil and alkaloid tetramethylpyrazine, also termed ligustrazine [3]. These active ingredients can exert distinct functions, either possessing neuroprotective role by elevating vasoactive molecules such as Nitric Oxide (NO) and Calcitonin Gene-Related Peptide (CGRP) [4,5], or regulating anti-inflammatory activity such as cytokines [6,7]. However, the role of these active ingredients in migraine and the associated mechanism are still not fully understood. Among these ingredients, phthalide compounds are regarded to have great therapeutic potential for treating migraine [7-9], in which Senkyunolide I (SEI, Figure 1) is a key component and shows effectiveness in migraine models. Oral administration of SEI can decrease pain threshold in a nitroglycerin-induced migraine model of rats [9]. When injected intraperitoneally in rats [10], the drug also suppresses the amplitude and frequency of Cortical Spreading Depression (CSD), the pathophysiological basis of migraine [1113]. The effectiveness of SEI on migraine models is associated with its vascular action in that the drug not only has the ability in regulating plasma 5-HIAA/5HT monoamine turnover rate [9], but also in reducing the elevated plasma concentrations of NO $[9,10]$ and CGRP induced by CSD in rats [10]. Whether SEI has neuronal action is still controversial. On one hand, SEI is unable to alter the elevated $\mathrm{NO}$ level in the nitroglycerin-induced migraine model of rats [9]. On the other hand, SEI attenuates oxygen-glucose deprivation/ reoxygenation-induced inflammation in microglial cells [7], which suggests a neuronal action. In light of these observations, it is worth clarifying if the mechanism of action of SEI in a migraine model is neuronal. Ligustrazine (Figure 1) is another active component of Chuanxiong Rhizoma. This component can strengthen antioxidative function by lowering methylenedioxyamphetamine (MDA) content and elevating Superoxide Dismutase (SOD) activity [14]. Ligustrazine also alleviates N-Methyl-D-Aspartate (NMDA) receptor-elicited injury of rat retinal ganglion cells both morphologically and functionally by reducing reactive oxidative stress (ROS) level, $\mathrm{Ca}^{2+}$ response to NMDA and inhibiting the L-type calcium channel [15], signals of which are key in migraine pathophysiology [16,17]. Given<smiles>CCC/C=C1\OC(=O)C2=C1CC[C@H](O)[C@H]2O</smiles>

Senkyunolide I<smiles>Cc1nc(C)c(C)nc1C</smiles>

Ligustrazine
Figure 1: Chemical structure of SEI and Ligustrazine. Wang et al. (C) All rights are reserved 


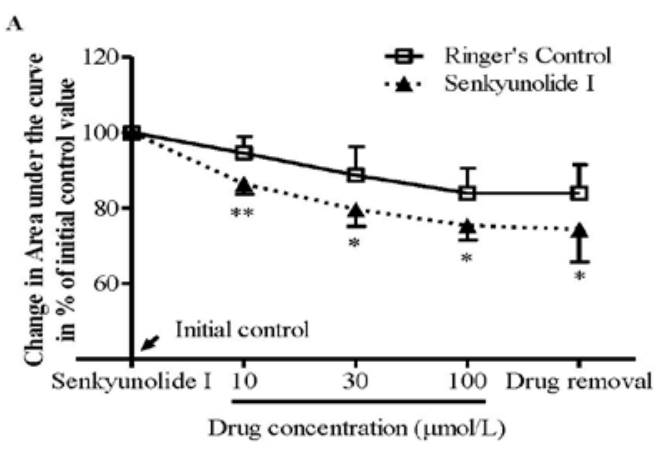

B

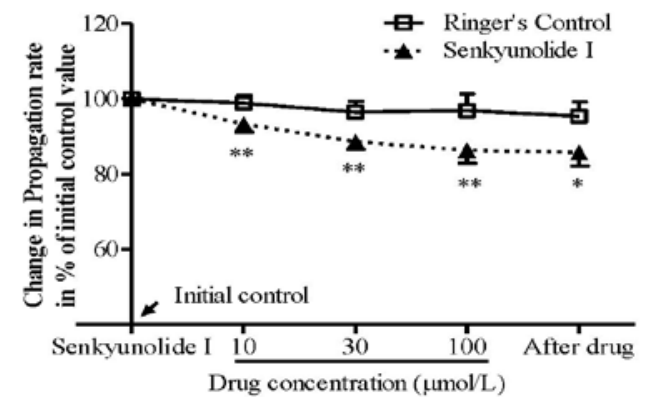

Figure 2: SEI suppressed both the magnitude $(A)$ and propagation rate $(B)$ of RSD induced by $\mathrm{KCl}^{+}$in the chick retina. Data was plotted as percentage of their initial levels and indicated as mean \pm SD. Data analyzed by MannWhitney $\mathrm{U}$ test for comparison between SEI $(n=6)$ and Ringer's control $(n=$ $6),{ }^{*} p<0.05,{ }^{* *} p \leq 0.01$

that both ROS and NMDA receptor are key in migraine mechanism as ROS can be induced by CSD in the trigeminal nociceptive system [18] and inhibition of NMDA receptor can block CSD in various migraine models $[16,19,20]$, we reason that ligustrazine may be also effective in suppressing CSD.

This study aimed to examine if both SEI and ligustrazine have anti-CSD effects via its neuronal action. A possible involvement of CGRP associated with SEI is also explored. To this end, an in vitro migraine model in chick retina was applied because this tissue is lack of blood vessels and Retinal Spreading Depression (RSD) has been previously used as a useful predictor of migraine for assessing antimigraine potential of drugs $[21,22]$.

\section{Materials and Methods}

Both SEI and ligustrazine were purchased from Pure One Biotechnology, China. Twenty-two male chicks (purchased at 1 day old, WuXi Yangjichang Ltd, Wuxi) were housed for at least a week before use (aged 8-28 days). The animal procedure was approved by the Ethical Review Panel of Soochow University under the agreement with Xi'an Jiaotong-Liverpool University (XJTLU) and performed in accordance with the associated guidelines. All efforts were made to minimize animal suffering to reduce number of animals used.

\section{RSD induction in chick retina}

Chick retina preparation and RSD induction were as that described previously [22]. Briefly, the posterior eyecup was positioned in a chamber. Unless otherwise stated, the chamber was perfused with Ringer's solution (concentrations in mM: $100 \mathrm{NaCl}, 6 \mathrm{KCl}, 1$
$\mathrm{MgSO}_{4}, 30 \mathrm{NaHCO}_{3}, 1 \mathrm{NaH}_{2} \mathrm{PO}_{4}, 1 \mathrm{CaCl}_{2}$ and 20 glucose; bubbled with $95 \% \mathrm{O}_{2}$ and $5 \% \mathrm{CO}_{2} ; \mathrm{pH} 7.3$ ) at $0.5 \mathrm{ml} /$ minute. The tissue was stabilized for at least 30 minutes before elicitation of the first RSD episode by ejection of $1 \mu \mathrm{l}$ of $0.1 \mathrm{M} \mathrm{KCl}$ at the edge of the eyecup and the temperature was kept constant at $32^{\circ} \mathrm{C}$.

\section{Intrinsic optical imaging}

The retina was illuminated for 25 Milli-Seconds (ms) using a high-power LED spotlight (625 nm peak wavelength, SLS-0307-A, Mightex; Pleasanton, USA) and the illumination was driven by a computer-controlled power supply (Sirius LED controller, SLCSA04-U; Mightex, Pleasanton, USA). The reflected light was simultaneously recorded with a monochrome camera (QIC-F-M-12, Qimaging, Media Cybernetics, Marlow, UK) used at a maximal spatial resolution. Image sequences were acquired at $1 \mathrm{~Hz}$ over a 3 -minute period, started as each RSD was elicited. Camera exposure and illumination were synchronized using an external trigger (TG5011, TTi, UK). Image Pro Plus software (IPP 7.0; Media Cybernetics, UK) was used for image acquisition, storage and analysis.

\section{Experimental design for drug testing}

Whether SEI and ligustrazine could alter RSD was examined with the following four groups. (i) SEI, concentration at 10,30 and $100 \mu \mathrm{M}$ $(\mathrm{n}=6)$; (ii) Ringer's solution was used as the control $(\mathrm{n}=6)$ for SEI; (iii) ligustrazine, concentrations at 20,60 and $200 \mu \mathrm{M}(\mathrm{n}=4)$; and (iv) DMSO (Sigma-Aldrich, Dorset, UK) at $0.01 \%, 0.03 \%$ and $0.1 \%$ was applied as the ligustrazine vehicle group in respective order (n =6). In each experiment, ten RSD episodes were induced, with each RSD followed by a 20-minute interval for tissue recovery. In order to minimize the animal use, two separate and consecutive RSDs was used for each of the following different tests: (i) initial Ringer's control; (ii) low concentration of drug or vehicle; (iii) medium concentration of drug or vehicle; (vi) high concentration of drug of vehicle; (v) posttreatment with Ringer's control (i.e. drug removal). For each test sequence, the perfusion medium was changed immediately after the end of the $2^{\text {nd }}, 4^{\text {th }}, 6^{\text {th }}$, and $8^{\text {th }}$ RSD recording when required, such that the preparation was adequately perfused with the proper drug or vehicle or Ringer's medium for the subsequent test. At end of RSD recording, the retina tissue was snap frozen in liquid nitrogen, transferred to $-80^{\circ} \mathrm{C}$ freezer for the subsequent measurement of CGRP level.

\section{Enzyme-linked immunosorbent assay (ELISA)}

Enzyme-Linked Immunosorbent Assay (ELISA) was applied for examining if SEI could alter release of CGRP during RSD. Protein was extracted from chick retina homogenate from both the above mentioned Ringer's control and SEI groups. Protein detection was carried out by following the manufacturer's instruction using the CGRP Enzyme Immunoassay kit (Bertin Pharma). Briefly, all retinas were rapidly homogenized within 15 seconds in $2 \mathrm{~N}$ acetic acid as 2 $\mathrm{ml} / 100 \mathrm{mg}$ tissue. The extract were heated to $90^{\circ} \mathrm{C}$ for 10 minutes, centrifuged at $10,000 \times \mathrm{g}$ for 30 minutes, dried for an hour and stored at $-80^{\circ} \mathrm{C}$. Immediately before assay, samples were reconstituted with enzyme immunoassay buffer and analyzed using a microplate reader (Synergy HT, BioTek, USA) for CGRP detection. CGRP levels are presented as the mean \pm SEM. 
A

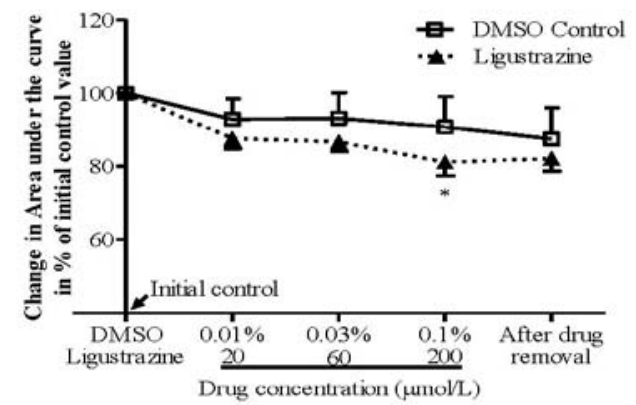

B

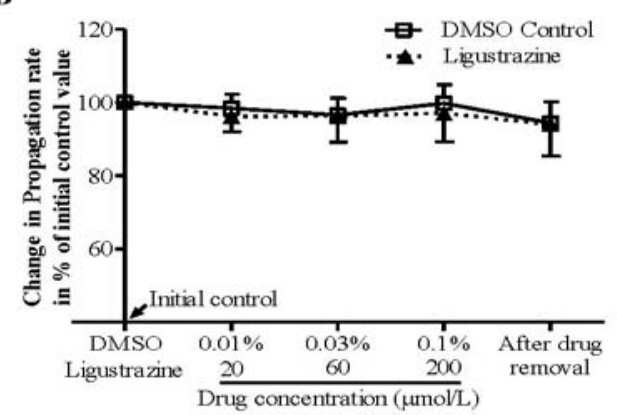

Figure 3: Ligustrazine suppressed the magnitude (A) but not the propagation rate $(B)$ of $\mathrm{RSD}$ induced by $\mathrm{KCl}$ in the chick retina. Data was plotted as percentage of their initial levels and indicated as mean \pm SD. Data analyzed by Mann-Whitney $U$ test for comparison between ligustrazine $(n=4)$ and DMSO control $(n=6),{ }^{*} p<0.05,{ }^{* *} p \leq 0.01$.

\section{Data presentation and statistical analysis}

For RSD data, as reported previously [20], for each 360-frame sequence, an Area of Interest (AOI) parallel to the RSD wave front was delineated manually. For each picture within the sequence, the gray levels of the pixels constitute in the AOI were averaged and plotted against the time as an indicator to characterize RSD. For each RSD wave, the area under the curve (AUC, gray level $x$ minute) of the transient cellular depolarization was calculated and used as an index of the magnitude of propagating RSD. Propagation rate was also calculated to reflect the degree of tissue excitability. The calculated values within each different test were averaged and all corresponding data were given as mean \pm SD in percentages of their respective baselines, that is, averaged value for the first two $\mathrm{K}^{+}$ stimuli. Two-tailed Mann-Whitney $\mathrm{U}$ test was used for comparison of the magnitude and propagation rate of RSD between the drug and respective control group. For ELISA data, statistical analyses were done using Graph Pad Prism and unpaired comparisons between the drug and control group were done using a one-tailed Mann-Whitney U-test.

\section{Results}

\section{SEI inhibited the magnitude and propagation rate of RSD}

We firstly examined the effects of SEI on RSD in the chick retina. In the Ringer's group, there was no significant change in both the magnitude and propagation rate over repeated RSD episodes throughout the experiment (Figure 2, $\mathrm{n}=6$ ). In SEI group, the drug suppressed the magnitude of RSD in a concentration dependent manner (Figure $2 \mathrm{~A}$ ). The magnitude was reduced to $86.4 \% \pm 2.8 \%$,
$69.6 \% \pm 4.5 \%$, and $75.4 \% \pm 3.8 \%$ of initial level at 10,30 and $100 \mu \mathrm{M}$ in respective order and this reduction was significant when compared with the Ringer's group ( $p=0.0087,0.0411$ and 0.0152 , in respective order, $\mathrm{n}=6$, Figure 2A). Similarly, the drug at all three concentrations tested also reduced RSD propagation rate to $93.4 \% \pm 2.2 \%, 88.6 \% \pm$ $2.1 \%$, and $86.4 \% \pm 3.4 \%$ in respective order (Figure $2 \mathrm{~B}$ ). This reduction was significant when compared with their respective Ringer's group $(\mathrm{p}=0.0087,0.0411$ and 0.0152 in order, $\mathrm{n}=6$, Figure $2 \mathrm{~B})$.

This inhibitory effect was persistent after the drug removal, and the magnitude and propagation rate of RSD was maintained at $74.4 \% \pm 8.6 \%$ and $85.8 \% \pm 3.6 \%$ of initial level, respectively, which was significant when compared with the control group $(\mathrm{p}=0.0411$ respectively, Figure 2).

\section{Ligustrazine suppressed the magnitude of RSD}

We further examined effects of ligustrazine on RSD in the chick retina. The results showed that DMSO at all concentrations tested did not alter both the magnitude and propagation rates of RSD (Figure $3, \mathrm{n}=6$ ). Ligustrazine at $20 \mu \mathrm{M}$ and $60 \mu \mathrm{M}$ did not alter RSD magnitude. However, at the highest concentration $(200 \mu \mathrm{M})$ tested, the drug reduced the magnitude of RSD to $81.3 \% \pm 4.8 \%$, and this reduction was significant when compared with respective vehicle group ( $\mathrm{p}=0.0381, \mathrm{n}=6$, Figure 3A). Differently, ligustrazine at all concentrations applied did not alter the propagation rate (Figure $3 \mathrm{~B}$ ).

\section{SEI does not alter retinal CGRP level during RSD}

As SEI suppressed both RSD magnitude and propagation rate and exhibited better efficacy than ligustrazine (Figures 3,4), only SEI was further considered for the subsequent examination of retinal CGRP levels after repeated RSD. The results showed that in the control group after repeated RSD was induced, chick retinal CGRP level was $0.93 \pm 0.21 \mathrm{ng} / \mathrm{g}$ tissue $(\mathrm{n}=6)$. In SEI group, the retinal CGRP level was slightly reduced to $0.67 \pm 0.07 \mathrm{ng} / \mathrm{g}$ tissue $(\mathrm{n}=6)$. However, this reduction was insignificant when compared to the control group (Figure 4).

\section{Discussion}

The key finding of this study is the inhibitory effect of SEI on the magnitude and propagation rate of RSD, suggesting a therapeutic potential of SEI for migraine treatment. This data extends a previous study in vivo that SEI suppresses the amplitude and frequency of CSD after intraperitoneal injection [10].

The mechanism of SEI on RSD is not clear. However, several possible explanations may account for this observation. Firstly, that the inhibitory effect was observed in the chick retina, a tissue that lack of blood vessels, suggests the neuronal action of SEI on RSD. Secondly, the effect of SEI on RSD is likely to be attributed to a reduced TNF- $\alpha$ as SEI can reduce the production of proinflammatory cytokines including TNF- $\alpha$ and inflammation-related enzymes that are stimulated by oxygen-glucose deprivation/reoxygenation in rats [10]. Supporting this notion comes from that TNF- a plays a key role in regulating CSD $[23,24]$ as in hippocampus, CSD increases TNF- $\alpha$ level [24] and this protein in turn increases the cortex susceptibility to CSD [23]. An alternative explanation to account for the suppressive effect of SEI on RSD is related to ROS. SEI is known to be an antioxidant as it decreases the levels of MDA and increases the activities of SOD to prevent the cerebral morphologic damage and brain edema 


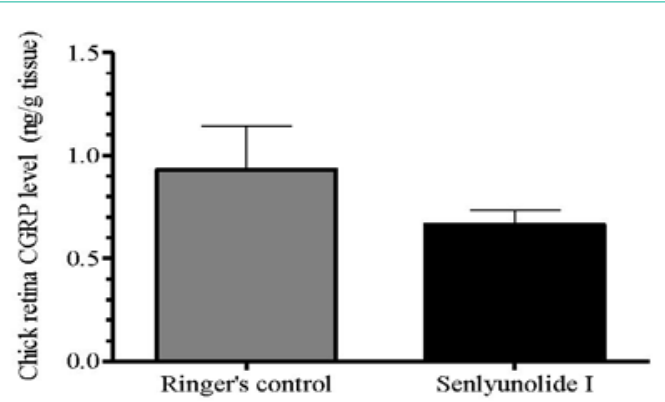

Figure 4: SEI slightly but not significantly reduced retinal CGRP peptide levels during RSD. Unpaired comparisons between SEI $(n=6)$ and Ringer's group $(n=6)$ were done using a one-tailed Mann-Whitney U-test.

in rats [4]; Whilst CSD induces ROS in trigeminal nociceptive system [18] and in microglial cells [23]. This raises the possibility that ROS as a link between SEI and its inhibition on RSD in the chick retina.

It is also possible that the action of SEI during RSD is associated with CGRP because increase in CGRP gene expression after CSD can be observed in various tissues including pia [25], neocortical slice [26] and cortex of rats [27] and SEI can reduce plasma CGRP contents induced by CSD in rats [10]. Interestingly, in this study, SEI only slightly but not significantly reduced retinal CGRP level in chick retina after RSD (Figure 4), which appears not to support the involvement of CGRP. However, one may argue that the inhibitory effect on RSD by SEI was persistent after SEI removal, suggesting a sufficient ligand-receptor binding. It is possible that the slight reduction of CGRP by SEI after RSD (Figure 4) may have been sufficient for modulating CGRP receptor activity in the chick retina, leading to RSD suppression. Additionally, a proportion of CGRP may have released into the chamber and perfused away during the last two RSD episodes after the drug removal, which may have limited a significant reduction of CGRP by SEI to be observed. Further investigation overcoming this limitation is necessary to address this possibility, in particular, unaltered brain CGRP contents by SEI is indeed shown in a nitroglycerin-induced migraine model [10].

Another finding of this study is that ligustrazine also suppressed RSD magnitude, which action is similar to that of SEI, but to a lesser extent (Figure 3). The reduction of RSD magnitude complements to a previous study that ligustrazine reduces neuropathic pain via its action on neuronal apoptosis in the rat spinal dorsal horn [28]. One plausible explanation to account for the inhibitory effect of ligustrazine on RSD is related to NMDA receptor/ $\mathrm{Ca}^{2+}$ signaling as these signals play key roles in migraine pathophysiology $[16,17]$. Ligustrazine is able to reduce ROS level and $\mathrm{Ca}^{2+}$ response to NMDA as well as inhibit the L-type calcium channel in rat retinal ganglion cells [15] and inhibition of NMDA receptor can block RSD [20]. Another explanation is related to ROS as ligustrazine can significantly strengthen antioxidative function by lowering MDA content and elevating SOD activity in mice [14].

In summary, this study demonstrates that both SEI and ligustrazine inhibit RSD, suggesting multiple active components in Chuanxiong Rhizoma may perform similar function in regulating key signals in migraine pathophysiology via neuronal mechanism. These two compounds may exert synergistic action for migraine therapy and this requires further investigation.

\section{Acknowledgement}

This work was funded by Wangwenli Charitable foundation.

\section{References}

1. Wang $\mathrm{YH}$, Hong $\mathrm{YL}$, Feng $\mathrm{Y}, \mathrm{Xu}$ DS, Liang $\mathrm{S}$, Lin $\mathrm{X}$, et al. Comparative pharmacokinetics of senkyunolide I in a rat model of migraine versus normal controls. European journal of drug metabolism and pharmacokinetics. 2012; 37: 91-97.

2. Yang $\mathrm{X}$, Zeng $\mathrm{X}, \mathrm{Wu} \mathrm{T}$. Chuanxiong preparations for preventing stroke. Cochrane Database Syst Rev. 2010.

3. Wang L, Zhang J, Hong Y, Feng Y, Chen M, Wang Y. Phytochemical and pharmacological review of da chuanxiong formula: a famous herb pair composed of chuanxiong rhizoma and gastrodiae rhizoma for headache. Evidence-based complementary and alternative medicine : eCAM. 2013; 2013.

4. Hu Y, Duan M, Liang S, Wang Y, Feng Y. Senkyunolide I protects rat brain against focal cerebral ischemia-reperfusion injury by up-regulating $p$-Erk1/2, Nrf2/HO-1 and inhibiting caspase 3. Brain Res. 2015; 1605: 39-48.

5. Feng Z, Lu Y, Wu X, Zhao P, Li J, Peng B, et al. Ligustilide alleviates brain damage and improves cognitive function in rats of chronic cerebral hypoperfusion. Journal of ethnopharmacology. 2012; 144: 313-321.

6. Ma Z, Bai L. The anti-inflammatory effect of Z-Ligustilide in experimenta ovariectomized osteopenic rats. Inflammation. 2012; 35: 1793-1797.

7. Hu YY, Wang Y, Liang S, Yu XL, Zhang L, Feng LY, et al. Senkyunolide I attenuates oxygen-glucose deprivation/reoxygenation-induced inflammation in microglial cells. Brain Res. 2016; 1649: 123-131.

8. Ma Q, Ma C, Wu F, Xiong YK, Feng Y, Liang S. Preparation and structural determination of four metabolites of senkyunolide I in rats using ultra performance liquid chromatography/quadrupole-time-of-flight tandem mass and nuclear magnetic resonance spectra. BMC complementary and alternative medicine. 2016; 16: 504.

9. Wang YH, Liang S, Xu DS, Lin X, He CY, Feng Y, et al. Effect and mechanism of senkyunolide I as an anti- migraine compound from Ligusticum chuanxiong. The Journal of pharmacy and pharmacology. 2011; 63: 261-266.

10. Duan M, Gu C, Dong X, Wang Y, Liang S, Feng Y. Pharmacodynamic Action of Senkyunolide I on Cortical Spreading Depression in Migraine Rats. Traditional Chinese Drug Research \& Clinical Pharmacology. 2013; 24: $217-$ 221.

11. Costa C, Tozzi A, Rainero I, Cupini LM, Calabresi P, Ayata C, et al. Cortical spreading depression as a target for anti-migraine agents. The journal of headache and pain. 2013; 14: 62

12. Eikermann-Haerter K, Ayata C. Cortical spreading depression and migraine. Current neurology and neuroscience reports. 2010; 10: 167-173.

13. Somjen GG. Aristides Leao's discovery of cortical spreading depression. J Neurophysiol. 2005; 94: 2-4.

14. Zhang C, Wang SZ, Zuo PP, Cui X, Cai J. Protective effect of tetramethylpyrazine on learning and memory function in D-galactoselesioned mice. Chin Med Sci J. 2004; 19: 180-184.

15. Luo X, YuY, Xiang Z, Wu H, Ramakrishna S, Wang Y, etal. Tetramethylpyrazine nitrone protects retinal ganglion cells against N-methyl-D-aspartate-induced excitotoxicity. Journal of neurochemistry. 2017.

16. Marrannes R, Willems R, De Prins E, Wauquier A. Evidence for a role of the $\mathrm{N}$-methyl-D-aspartate (NMDA) receptor in cortical spreading depression in the rat. Brain Res. 1988; 457: 226-240.

17. Pietrobon D. Calcium channels and migraine. Biochimica et biophysica acta. 2013; 1828: 1655-1665

18. Shatillo A, Koroleva K, Giniatullina R, Naumenko N, Slastnikova AA, Aliev $\mathrm{RR}$, et al. Cortical spreading depression induces oxidative stress in the trigeminal nociceptive system. Neuroscience. 2013; 253: 341-349.

19. Peeters M, Gunthorpe MJ, Strijbos PJ, Goldsmith P, Upton N, James 
MF. Effects of pan- and subtype-selective N-methyl-D-aspartate receptor antagonists on cortical spreading depression in the rat: therapeutic potential for migraine. The Journal of pharmacology and experimental therapeutics. 2007; 321: 564-572.

20. Wang M, Chazot PL, Ali S, Duckett SF, Obrenovitch TP. Effects of NMDA receptor antagonists with different subtype selectivities on retinal spreading depression. British journal of pharmacology. 2012; 165: 235-244.

21. Wiedemann M, Lyhs B, Bartels JP, Sieber M. The pharmacological control of neuronal excitability in the retinal spreading depression model of migraine Current medicinal chemistry. 2012; 19: 298-302.

22. Wang M, Li Y, Lin Y. GABAA receptor alpha2 subtype activation suppresses retinal spreading depression. Neuroscience. 2015; 298: 137-144.

23. Grinberg YY, Dibbern ME, Levasseur VA, Kraig RP. Insulin-like growth factor-1 abrogates microglial oxidative stress and TNF-alpha responses to spreading depression. Journal of neurochemistry. 2013; 126: 662-672.

24. Kunkler PE, Hulse RE, Kraig RP. Multiplexed cytokine protein expression profiles from spreading depression in hippocampal organotypic cultures.
Journal of cerebral blood flow and metabolism: official journal of the International Society of Cerebral Blood Flow and Metabolism. 2004; 24: 829839.

25. Wahl M, Schilling L, Parsons AA, Kaumann A. Involvement of calcitonin gene-related peptide (CGRP) and nitric oxide (NO) in the pial artery dilatation elicited by cortical spreading depression. Brain Res. 1994; 637: 204-210.

26. Tozzi A, de lure A, Di Filippo M, Costa C, Caproni S, Pisani A, et al. Critical role of calcitonin gene-related peptide receptors in cortical spreading depression. Proceedings of the National Academy of Sciences of the United States of America. 2012; 109: 18985-18990.

27. Wang $Y$, Tye AE, Zhao J, Ma D, Raddant AC, Bu F, et al. Induction of calcitonin gene-related peptide expression in rats by cortical spreading depression. Cephalalgia: an international journal of headache. 2016.

28. Leng YF, Gao XM, Wang SX, Xing YH. Effects of tetramethylpyrazine on neuronal apoptosis in the superficial dorsal horn in a rat model of neuropathic pain. Am J Chin Med. 2012; 40: 1229-1239.
Austin J Pharmacol Ther - Volume 5 Issue 1 - 2017

ISSN: 2373-6208 | www.austinpublishinggroup.con Wang et al. (C) All rights are reserved
Citation: Li YL, Wang Y and Wang M. Both Senkyunolide I and Ligustrazine Suppress Retinal Spreading Depression. Austin J Pharmacol Ther. 2017; 5(1).1091. 